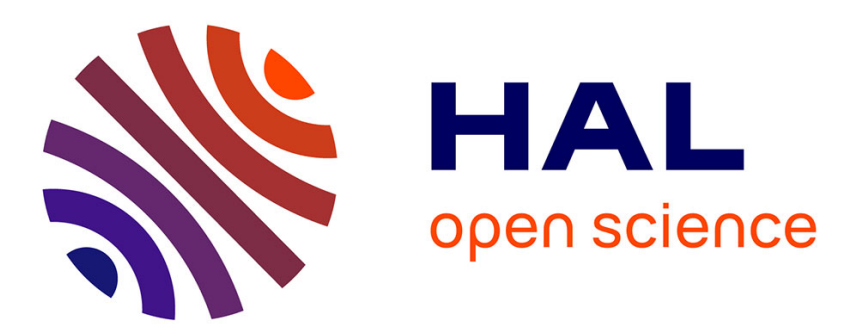

\title{
Assignment of Roles and Channels for a Multichannel MAC in Wireless Mesh Networks
}

Fabrice Theoleyre, Benoit Darties, Andrzej Duda

\section{To cite this version:}

Fabrice Theoleyre, Benoit Darties, Andrzej Duda. Assignment of Roles and Channels for a Multichannel MAC in Wireless Mesh Networks. International Conference on Computer Communications and Networks (ICCCN), 2009, United States. pp.10.1109/ICCCN.2009.5235224. hal-00618224

\section{HAL Id: hal-00618224 \\ https://u-bourgogne.hal.science/hal-00618224}

Submitted on 1 Sep 2011

HAL is a multi-disciplinary open access archive for the deposit and dissemination of scientific research documents, whether they are published or not. The documents may come from teaching and research institutions in France or abroad, or from public or private research centers.
L'archive ouverte pluridisciplinaire HAL, est destinée au dépôt et à la diffusion de documents scientifiques de niveau recherche, publiés ou non, émanant des établissements d'enseignement et de recherche français ou étrangers, des laboratoires publics ou privés. 


\title{
Assignment of Roles and Channels for a Multichannel MAC in Wireless Mesh Networks
}

\author{
Fabrice Theoleyre, Benoit Darties and Andrzej Duda \\ Grenoble Informatics Laboratory, Grenoble, France \\ Email:\{darties, theoleyr, duda\}@imag.fr
}

\begin{abstract}
A multichannel MAC improves throughput in wireless mesh networks by multiplexing transmissions across orthogonal channels. In this paper, we propose an efficient way for constructing the wireless mesh structure associated with Molecular MAC, a multichannel MAC layer for efficient packet forwarding. Molecular MAC outperforms other classical approaches, but requires a specific structure for efficient operation. First, we propose a centralized protocol that provides an upper bound for constructing such a molecular structure through a MILP (Mixed Integer Linear Programming) formulation that maximizes network capacity. Then, we present two distributed self-stabilizing heuristic protocols derived from the protocols for constructing respectively a Maximum Independent Set and a Spanning Tree. We compare the performance of the proposed protocols in terms of network capacity and route length.
\end{abstract}

\section{INTRODUCTION}

We consider wireless mesh networks that use IEEE 802.11 wireless LANs for interconnecting mesh routers. When mesh routers use the legacy IEEE 802.11 networks with a single interface, performance of packet forwarding quickly degrades with the number of hops due to channel contention and spatial problems such as hidden, exposed, masked, and blocked nodes [1]. We can observe that the capacity of a wireless mesh network strongly depends on the ability of nearby mesh routers to communicate in parallel, which is only possible if neighbor routers, which may interfere, use different channels.

One way of improving performance is to use multiple non-overlapping channels. When a mesh router has several radio interfaces, it can tune them to different channels and simultaneously communicate without interference. The main problem is thus to assign channels to interfaces in a way that maximizes network capacity. If the mesh network is relatively small, we can use a global optimization approach [2], [3]. However, for networks with an increasing number of mesh routers, distributed approaches fit better [4], [5], [6]. Hierarchical clustered networks can use one radio interface for intra-cluster communications and another one for intercluster transmissions[7]. At the same time, spontaneous mesh networks may include nodes with only one single interface. In this case, nodes can dynamically switch channels so that interfering neighbor nodes simultaneously transmit on orthogonal channels. Molecular MAC proposes to organize the mesh network according to the molecular analogy [8]: it divides the network into atoms with nucleus nodes operating on fixed channels and electrons that dynamically switch channels between neighbor nuclei. Recent work [9] has shown that Molecular MAC largely outperforms standard
IEEE 802.11 networks and other approaches such as MMAC (Multi-Channel MAC) [10]. However, the Molecular MAC proposal did not deal with the construction of a molecular structure: the authors left the problem to a future work. In this paper, we address the problem of constructing such a molecular structure, e.g. electing nucleus nodes and assigning channels to interfaces.

The contribution of this paper is twofold. First, we formulate the problem of electing nucleus nodes and assigning channels as a generic MILP (Mixed Integer Linear Programming) problem. Its solution leads to the optimal assignment of roles (nucleus or electron) and channels in a spontaneous mesh network. Second, we propose two distributed protocols to solve the problem in a large mesh network and evaluate their performance through simulations. We show that MILP formulation and its optimal solution provide a suitable metric to compare the performance of different heuristics. Finally, our approach and problem formulation represent a solid basis for addressing many multichannel MAC problems.

After briefly presenting Molecular MAC, we will formulate the assignment problem. Then, Section IV concerns finding the optimal solution and Section V describes two proposed distributed protocols. We evaluate their performance and conclude after discussing the related work.

\section{Molecular MAC OVERView \& Motivations}

The present paper aims at defining distributed protocols for constructing the molecular architecture required by Molecular MAC. Thus, we propose to start with a brief overview of Molecular MAC and its operation. Molecular MAC achieves efficient packet forwarding over multiple hops through multiplexing parallel transmissions over multiple channels. It solves the deafness problem without using a fixed signaling channel (if an access method reserves a channel for signaling, there are less resources available for data transmission) nor periodical rendezvous (a rendezvous increases overhead and requires one form of temporal synchronization between mesh routers).

IEEE 802.11 wireless networks work fairly well in the infrastructure mode and with small modifications to their access method, they can achieve fair distribution of bandwidth to each client [11]. However, several key problems arise in multihop networks, as highlighted by Chaudet et al. [1]. Molecular MAC proposes to extend IEEE 802.11 to efficiently deal with packet forwarding in multihop networks.

Since IEEE 802.11 access method works well in single-hop networks, Molecular MAC divides a wireless mesh network 
into spatially distributed atoms so that each atom uses a fixed channel different from its neighbors. An atom is composed of a nucleus and electrons. A nucleus chooses a channel for its atom and sticks to the channel all the time. Nodes at the border of atoms have the role of electrons bonding neighboring atoms: they forward traffic between atoms by dynamically switching their channels to communicate with neighboring nuclei. Two electrons do not directly communicate, because otherwise they may experience deafness when an electron tries to send a frame to another electron that is listening to another channel. There is no deafness related to a nucleus - it operates all the time on the same channel. Nodes participate in neighborhood discovery to detect new nodes and integrate them into the molecular structure with a suitable role, either nucleus or electron. Figure 1 illustrates this view. Mesh routers $N$ and $\mathrm{M}$ are nuclei of two atoms bonded by two electrons $\mathrm{B}$ and $\mathrm{C}$.

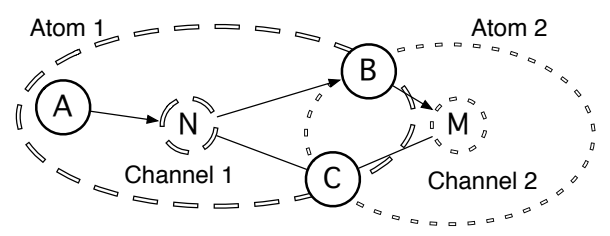

Fig. 1. Two atoms sharing two electrons

An electron must be able to receive packets from its neighboring nuclei. To achieve this, an electron has to explicitly request a data frame from its nucleus with a special control frame, a pull that acts a little bit like a Clear To Send (CTS) frame, but the CTS reservation is only reception oriented. Finally, each nucleus piggybacks the list of pending destinations in each data frame so that the electrons know when they need to request a frame through sending a pull. A nucleus maintains this activity by sending an empty data frame fast notification.

The molecular architecture is similar to clustering, each nucleus being a clusterhead and each electron being agateway. However, we can highlight the following key differences:

1) communication only takes place over nucleus-electron or electron-nucleus links. However, the network must stay connected;

2) the molecular structure aims at minimizing interference while clustering minimizes the number of clusters;

3) each nucleus uses a static channel for its transmissions. It should maximize network capacity.

Molecular MAC reduces the number of links in the mesh, however it improves network throughput in a significant way and results in using almost the shortest routes in practice [9].

In this paper, we propose protocols for constructing an efficient mesh molecule. Previously, our simulations have shown that Molecular MAC results in efficient packet forwarding. Our objective here is to only deal with the problem of mesh construction through role assignment and channel selection and not with more MAC oriented aspects already studied elsewhere [9]. We present below the optimal LP formulation of the problem and heuristics for distributively assigning roles.

\section{PROBLEM FORMULATION AND NOTATION}

We consider the problem of constructing a wireless mesh network that follows the molecule approach. First, we introduce notation-we model the network as an undirected graph $G=(\mathcal{V}, \mathcal{E})$, where $\mathcal{V}$ is the set of nodes and $\mathcal{E}$ the set of edges corresponding to two nodes able to directly communicate. We adopt the following notation:

- $n=|\mathcal{V}|$ defines the number of nodes in the mesh network,

- $N(u)$ is the set of neighbor nodes of $u$ with cardinality $\Delta(u)=|N(u)|$

- $\{u, v\}$ denotes the edge between vertices $u$ and $v$, i.e. $\{u, v\} \in \mathcal{E}$

- $B W$ denotes the radio bandwidth,

- $\mathrm{CH}$ is the set of all available channels and $n b C H=$ $|\mathrm{CH}|$ their number (IEEE 802.11a has for instance 12 orthogonal channels).

We need to assign a role to each node (a nucleus or an electron) so that the resulting molecule has the following properties:

1) a node can communicate with any other node via multihop forwarding,

2) only nuclei and electrons can communicate with each other, i.e. we exclude communications between two electrons or two nuclei,

3) the capacity of the network should be maximal. In particular, two neighboring atoms, which can interfere, need to use different channels.

The construction of the mesh molecule is closely related to a well-known graph structure problem-a Weakly Connected Dominating Set (WCDS) [12] formally defined by a set $D \subseteq$ $V$ such as :

$$
\begin{aligned}
& \forall u \in\{\mathcal{V}-D\}, \quad \exists v \in D / v \in N(u) \\
& \mathcal{G}\left(\mathcal{V}, \mathcal{E}^{\prime}\right) \text { connected/ } \quad \mathcal{E}^{\prime}=\{(u, v) / u \in D, v \in \mathcal{V}\}
\end{aligned}
$$

The set of nuclei form a restricted WCDS, i.e. a WCDS in which the graph weakly induced by the edges (nucleus,electron) forms a connected set. Formally, we transform the second property of Eq. 2 into:

$$
\mathcal{G}\left(\mathcal{V}, \mathcal{E}^{\prime}\right) \text { connected } / \mathcal{E}^{\prime}=\{(u, v) / u \in D, v \in\{\mathcal{V}-D\}\}
$$

In other words, we remove radio links between two nuclei, because they operate in a molecular mesh at different channels. Thus, they may only communicate through neighboring electrons. In addition, we aim at constructing a restricted WCDS that maximizes network throughput multiplexing transmissions across different channels.

\section{OPtimal MOLECULE CONSTRUCTION AND CHANNEL ASSIGNMENT}

We start with a MILP formulation of the problem that will give us an upper bound for comparing performance of proposed distributed protocols.

Our objective is to find a suitable role for each node (nucleus or electron) and assign channels to nuclei. Since many assignments are possible, we aim at finding the allocation that 
maximizes network throughput. Alazemi et al. proposes to define the objective as a sum of radio transmissions [13]. As the authors state, this privileges single hop flows thus leading to a suboptimal allocation for many applications. They also propose to maximize the minimum utilization of one particular channel. Since such a macroscopic metric does not capture bottlenecks and different route lengths, it does not correspond to real network capacity. Thus, we maximize the guaranteed network throughput: we assume that all possible flows are simultaneously active and we maximize the minimum throughput allocated to each flow. This metric clearly reflects the capacity of the network to forward high load.

\section{MILP Formulation}

We assign a role to each node $u \in \mathcal{V}$ represented by variable $r(u) \in\{0,1\}$ with value 1 if $u$ is a nucleus and 0 otherwise. For each pair $(u, c)$, where $u \in \mathcal{V}$ and $c \in[1, n b C H]$, variable $C H(u, c) \in\{0,1\}$ indicates if node $u$ uses channel $c$ $(C H(u, c)=1)$ or not $(C H(u, c)=0)$. Our performance objective is to maximize $T_{m i n}$, the minimum throughput allocated to each flow. Since all the nodes communicate with each other, there are $n(n-1)$ flows in the network.

MILP formulation requires to define a set of variables $T(u, v, d)$ that correspond to traffic transmitted by $u$ through radio link $\{u, v\}$ to destination $d$ for each triplet $(u, v, d) \mid\{u, v\} \in \mathcal{E}, d \in V$. As our formulation also requires traffic decomposition into different channels, we need to introduce additional variables $T_{c h}(u, v, c, d)$ that represent the portion of traffic going through link $(u, v)$ to $d$ on channel $c$.

1) One channel per nucleus: First, we assign exactly one channel to a nucleus and none to an electron:

$$
\forall u \in \mathcal{V}, \quad \sum_{c=0}^{n b C H} C H(u, c) \leq r(u)
$$

2) A link between an electron and a nucleus: We can only use a link if and only if its endpoints have different roles. Its capacity (the sum of $T(u, v, d)$ over all destinations $d$ ) is zero if both endpoints are nuclei (Eq.5) or electrons (Eq.6):

$$
\begin{aligned}
& \forall\{u, v\} \in \mathcal{E}, \\
& r(u)+r(v)+\frac{1}{B W} \sum_{d \in V}(T(u, v, d)+T(v, u, d)) \leq 2 \\
& \frac{1}{B W} \sum_{d \in V}(T(u, v, d)+T(v, u, d)) \leq r(u)+r(v)
\end{aligned}
$$

3) Flow conservation: Eq. 7 and 8 express the flow conservation law: traffic for $d$ coming from $u \neq d$ is equal to the sum of traffic for $d$ forwarded by $u$ and traffic generated by $u$ to $d\left(=T_{\min }\right)$. A destination node must receive exactly $(n-1) \cdot T_{\min }$ total traffic units sent by $(n-1)$ other nodes:

$$
\begin{aligned}
& \forall u, d \in \mathcal{V}, d \neq u, \\
& \sum_{v \in N(u)} T(u, v, d)=T_{\min }+\sum_{v \in N(u)} T(v, u, d) \\
& \forall u \in \mathcal{V}, \quad(n-1) \cdot T_{\min }=\sum_{v \in N(u)} T(v, u, u)
\end{aligned}
$$

4) Radio capacity: All interfering radio links need to share radio bandwidth. Obviously, the sum of traffic over all channels corresponds to the whole traffic (Eq. 9). Besides, all radio links that use the same channel must share channel capacity (Eq. 10). $I(e)$ represents the list of links interfering with $e$ that can be directly extracted from the conflict graph (cf. [14], we associate one vertex to each radio link in the conflict graph; an edge exists in the conflict graph if two corresponding radio links interfere in the original network):

$$
\begin{aligned}
& \forall u \in \mathcal{V}, \quad \sum_{c \in \text { Channels }}\left(T_{c h}(u, v, c, d)\right) \leq T(u, v, d) \\
& \forall e \in \mathcal{E}, \forall c \in C H, \sum_{(u, v) \in I(e)} \sum_{d \in V}\left(T_{c h}(u, v, c, d)\right) \leq B W
\end{aligned}
$$

5) Optimizing atom capacity: All links belonging to an atom share its bandwidth $B W$ (Eq. 11). The constraints are obvious if $u$ is a nucleus. If $u$ is an electron, it cannot receive more than BW, even if it is adjacent to several nuclei because of time sharing mechanisms to switch between frequencies.

$$
\forall u \in \mathcal{V}, \quad \sum_{v \in N(u)} \sum_{d \in V}(T(u, v, d)+T(v, u, d)) \leq B W
$$

6) Improvement: Optional inequalities (Eq. 12) accelerate the MILP resolution by stating that each nucleus is adjacent to at least one electron and reciprocally:

$$
\forall u \in \mathcal{V}, \quad 1 \leq r(u)+\sum_{v \in N(u)} r(v) \leq \Delta(u)
$$

7) MILP Objective: We aim at maximizing the minimum throughput, i.e. $\max T_{\min }$.

\section{Distributed PROTOCOLS FOR ROLE AND CHANNEL ASSIGNMENT}

In this section, we propose two distributed protocols for constructing a molecule. In the first strategy, we adopt a pure localized approach: a node decides to become a nucleus when no other neighbors become nuclei. Thus, the first approach builds on the construction of a Maximum Independent Set (MIS). In the second strategy, we propose to construct a self-stabilizing spanning tree. By coloring appropriate nodes, we consequently construct a WCDS that achieves desired properties.

\section{A. Maximum Independent Set}

The simplest localized protocol consists of assigning the nucleus role to the nodes that do not have any neighboring nodes that become nuclei. To avoid making decisions synchronously, we force each node to start a timer for a random duration. After a timeout, a node becomes nucleus if none of its neighbors has become a nucleus. Symmetrically, the neighbors of a nucleus automatically become electrons. The resulting graph forms a Maximum Independent Set by definition: no pair of neighboring nuclei exists, each electron is a neighbor of at least one nucleus, and a node is either an electron or a nucleus. Nuclei are dominating nodes in the MIS terminology. 
The first advantage of this approach is its locality property: the protocol quickly converges in finite time (more precisely, it is bounded by the timeout value). Thus, nodes can construct a MIS only with one hello packet transmitted as a broadcast.

In addition to obtaining the right role assignment, we need to minimize the number of nuclei to reduce interference between neighboring nuclei: a graph containing a smaller number of nuclei reduces the probability that two atoms use the same channel, which may result in interference. Thus, we improve the network capacity by limiting interference.

However, the main drawback of this approach relates to connectivity: a MIS does not lead to a connected graph in all cases. Consider for example a chain of four nodes: the two extremity nodes become nuclei and the other nodes are electrons-we obtain two disconnected atoms. This structure forms a MIS, but the graph weakly induced by the edges (nucleus,electron) is not always connected. Such cases occur even more frequently in networks with low density. If we have a dense random graph, the MIS will be connected with high probability: several paths exist between a pair of nodes and the probability that all paths do not exist in the restricted WCDS structure is small. To explore this issue, we have simulated random mesh networks and verified that the size of the largest connected component quickly reaches the size of the largest component in the original graph (Fig. 2).
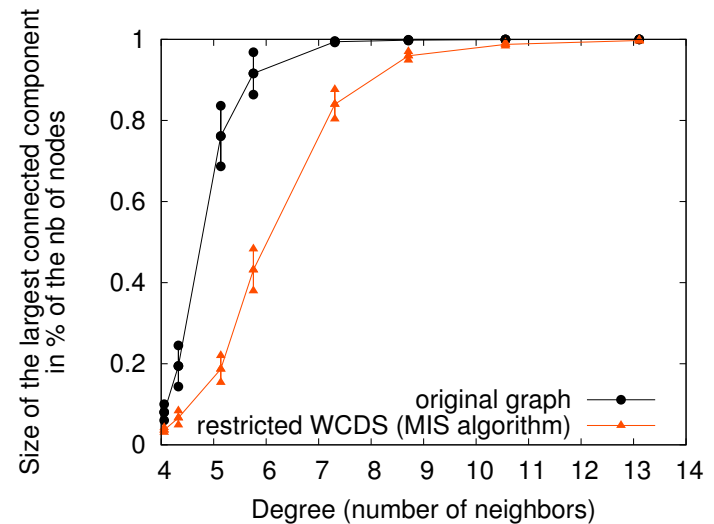

Fig. 2. Size of the largest connected component in MIS for random mesh networks

\section{B. Self-stabilizing spanning tree}

A spanning tree is a well-known structure to maintain connectivity in networks. We propose to construct a spanningtree with desired properties in three steps.

First, we construct the shortest path spanning tree rooted at the node with the smallest identifier in the network. By propagating the minimum known identifier and its distance in hops in hello packets, each node can update the information so that the network only maintains one spanning tree. The root generates a strictly increasing sequence number in each of its hello packets. Its neighbors forward the sequence number as is thus guaranteeing loop detection when the information about the distance to the root becomes obsolete.
Second, we assign the nucleus role to each node with an even depth in the tree and the electron role otherwise. Hence, we obtain a connected structure: each node has a path alternating nuclei and electrons towards the root.

Third, since we aim at minimizing interference, we must limit the number of interfering atoms, which is equivalent to minimizing the number of nuclei. Thus, we propose a simple pruning rule to eliminate redundant nuclei: a nucleus can safely become electron if simultaneously:

1) it has no child in the tree,

2) one neighbor with a smaller id is nucleus (to break a possible tie),

3) it has no neighbor with a smaller id with the same depth.

The proposed protocol is distributed: each node only needs the information transmitted in hello packets by its neighbors to decide which role it should adopt. Moreover, it converges in $O(D), D$ being the network diameter.

Besides, the protocol is self-stabilizing converging to a valid state after a finite number of steps: the root of the spanning tree sends hello packets with an increasing sequence number. If it ceases its operation, the sequence number does not increase and all the nodes will choose another root with the new smallest identifier after a timeout. Similarly, sequence numbers avoid the appearance of loops, which is vital in mesh networks prone to failures or topology changes.

Finally, the spanning tree tends to improve the capacity by keeping a large number of radio links in the molecule (we only remove radio links among nodes with the same depth). Besides, pruning some nuclei tends to limit interference among atoms. Simulations corroborate the efficiency of the molecule construction from the network throughput point of view.

\section{Channel assignment}

In our distributed approach, we first propose to construct a molecule and then greedily assign channels to each nucleus. A nucleus asks its electrons to provide information about channel activity they can measure. Electrons scan all the channels, measure their activity expressed as the number of transmitted packets and report them to their nucleus. A nucleus proceeds in the following manner to collect statistics:

1) it sends a broadcast with a channel activity request to all neighboring electrons

2) the electrons scan the channels and report channel activity in a unicast frame to the nucleus

3) the nucleus eventually repeats its request in a unicast frame to non-replying electrons (to be robust to packet transmission errors). When it obtains all replies, it chooses a channel according to the following preference:

a) if some channels are inactive for all electrons, randomly choose one,

b) otherwise, choose the channel that minimizes the maximum activity for all the members of an atom.

Nodes assign channels after deciding their roles, because a node should take into account the activity in its vicinity before choosing the best suitable channel. Moreover, the activity 


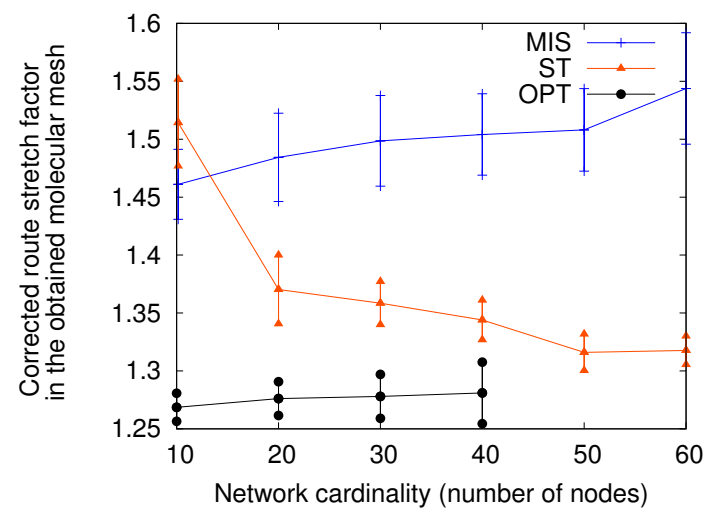

Fig. 3. Route stretch factor for varying network cardinality

depends on the role: an electron will transmit packets on each channel chosen by its neighboring nuclei.

\section{PERFormanCE EVALUATION}

We have simulated the proposed protocols in WsNet[15] using the COIN-CBC LP library [16]. We randomly place nodes in a simulation area. Nodes use the IEEE 802.11a network interface to communicate with each other with the radio range of 10 units and the interference range of 30 units. WsNet assumes the free-space model for radio propagation. By default, the mesh network is composed of 50 nodes with the average number of neighbors of 10 . We adjust the simulation area to obtain a given density.

The results correspond to statistics averaged over 10 different simulations of 240 seconds. The graphs present averaged values with $95 \%$ confidence intervals. We compare the performance of the MILP formulation (OPT), the Maximum Independent Set protocol (MIS), and the self-stabilizing Spanning Tree (ST) (cf. Section V).

\section{A. Route stretch factor}

First, we measure the route stretch factor: the ratio of the route lengths in a molecular mesh and in the original graph (cf. Fig. 3). A stretch factor of 1 means that only the shortest routes are used. For MIS, we discard isolated nodes since the stretch factor would become infinite in this case. Thus, we tend to under-estimate the real stretch factor for MIS. We do not have any result for the OPT strategy in networks with more than 40 nodes since MILP does not find a feasible and optimal assignment after a reasonable computing time (i.e. less than 2 hours). We can note that the OPT strategy results in using short routes. Thus, we can legitimately consider that a small stretch factor will optimize the global network throughput. ST uses longer routes than OPT, but the difference tends to decrease when the number of nodes increases: the spanning tree achieves to find short routes. On the contrary, MIS discovers longer routes when the network cardinality increases: two electrons can separate two nuclei thus forcing longer routes.

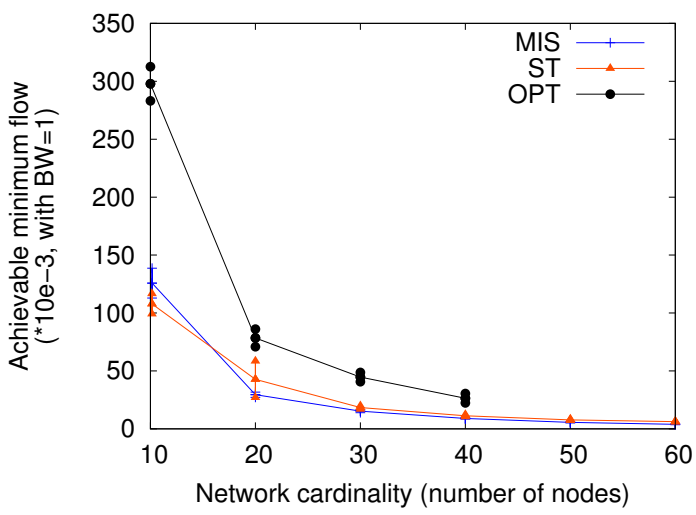

Fig. 4. Capacity (min-flow objective) for varying network cardinality

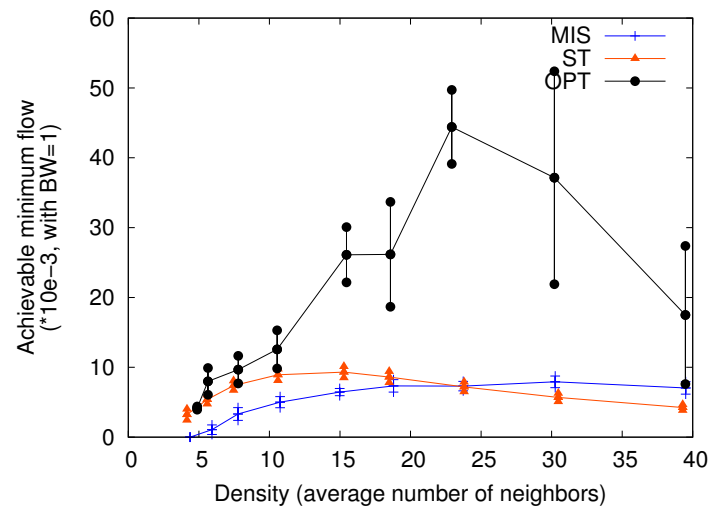

Fig. 5. Capacity (min-flow objective) for varying density

\section{B. Capacity through channel diversity}

Then, we measure network throughput defined as $T_{\min }$ in the MILP formulation. Thus, for the ST and MIS strategies, we run the MILP formulation with already assigned roles and channels to obtain the minimum flow for each molecular topology.

We first measure the impact of the network cardinality (cf. Fig. 4) while maintaining constant density. Obviously, the OPT strategy gives us an upper bound. Moreover, we can note that MIS and ST achieve a much lower throughput: since they are distributed, they cannot optimize the global throughput as the OPT strategy does.

Second, we consider the impact of the density on the network capacity (cf. Fig. 5) while maintaining the number of nodes constant. We can observe that the capacity first increases with the density since the routes become shorter and consume less bandwidth. Then, it decreases, because the radio spatial reutilization decreases. We can also note that for high density, MIS performs better than ST: MIS efficiently prunes the network thus reducing the number of interfering nuclei. On the contrary, ST is more suitable for low density, since it maintains a good route length stretch factor helping to improve throughput. 


\section{RELATED WORK}

Considering multichannel wireless networks has received large attention recently: they multiplex communications over orthogonal channels thus improving the global throughput. One approach consists of dynamically switching channels on a per packet basis [10], [17], [8]. In MMAC [10], all nodes periodically reserve the channel for data transmission on a control channel. In SSCH [17], each node chooses a pseudorandom channel sequence to follow and exchange packets with neighbors when they are on the same channel.

The second approach assigns a fixed channel to each radio link when the mesh network includes nodes with multiple radio interfaces. In this case, two nodes are able to communicate iff one of their radio interfaces uses the same channel. Alazemi et al. [13] present a MILP formulation of the multiradio multichannel assignment closely related to the problem presented here. The authors try to linearly formulate all the limiting constraints, e.g. the number of channels used per node. However, they do not optimize the global traffic, but rather channel diversity as explained in Section IV, which does not lead to the optimal network capacity. Marina et al. [6] propose to greedily allocate channels when the assignment is achievable. Other authors further enrich their algorithm [18]. This strategy is centralized and complex to transform into a distributed protocol. Avallone et al. [4] propose a more sophisticated strategy: they first compute a flow rate in the network without taking into account interference. Then, they extract groups and greedily assign orthogonal channels to groups with interfering links.

Other approaches propose distributed protocols for multichannel networks. Ramachandran et al. [2] try to estimate interference to allocate orthogonal channels to interfering edges. Nguyen et al. [3] assign channels for multicast mesh networks: the case is less complex than the unicast problem, because a node just receives traffic on a channel and forwards it as a broadcast to all its children in the multicast tree. Moreover, the authors independently optimize the multicast tree and the channel assignment algorithm thus resulting in a suboptimal solution. Naveed et al. [5] divide the network into clusters and allocate one channel per cluster thus limiting the route length by creating a spanner.

\section{CONCLUSIONS AND FUTURE WORK}

In this paper, we have proposed two distributed protocols for constructing the structure required by Molecular MAC. We have also formulated a MILP problem that provides an upper bound we can achieve to maximize the network throughput. In a more general way, the constraints in the MILP formulation are very common in multichannel mesh networks and can be further used for considering optimal assignment of channels for this type of networks. We are currently working on making the MILP formulation distributed so that the structure can be locally optimized. Besides, we also aim at integrating sophisticated interference measurement protocols to support our channel assignment protocol so that a mesh network selfadapts to interference and traffic variation.

\section{ACKNOWLEDGMENTS}

This work was partially supported by the European Commission project WIP under contract 27402, the French Ministry of Research project AIRNET under contract ANR-05RNRT-012-01, and France Telecom contract 3-04-36.

\section{REFERENCES}

[1] C. Chaudet, D. Dhoutaut, and I. Guerin Lassous, "Performance issues with IEEE 802.11 in ad hoc networking," IEEE Communications Magazine, vol. 43, no. 7, 2005.

[2] K. N. Ramachandran, E. M. Belding, K. C. Almeroth, and M. M. Buddhikot, "Interference-aware channel assignment in multi-radio wireless mesh networks," in INFOCOM. Spain: IEEE, 2006.

[3] H. L. Nguyen and U. T. Nguyen, "Channel assignment for multicast in multi-channel multi-radio wireless mesh networks," Wireless Communications and Mobile Computing, 2008.

[4] S. Avallone and I. F. Akyildiz, Ian F.ildiz, "A channel assignment algorithm for multi-radio wireless mesh networks," Computer Соттиnications, vol. 31, no. 7, 2008.

[5] A. Naveed, S. S. Kanhere, and S. K. Jha, "Topology control and channel assignment in multi-radio multi-channel wireless mesh networks," in MASS. Italy: IEEE, 2007.

[6] M. K. Marina and S. R. Das, "A topology control approach for utilizing multiple channels in multi-radio wireless mesh networks," in BROADNETS. USA: IEEE, 2005.

[7] J. Zhu, S. Roy, X. Guo, and W. S. Conner, Advances in Pervasive Computing and Networking. Springer, 2005, ch. Maximizing Aggregate Throughput in 802.11 Mesh Networks with Physical Carrier Sensing and Two-Radio Multi-Channel Clustering.

[8] M. Nassiri, F. Theoleyre, M. Heusse, and A. Duda, "Molecular architecture for autonomic wireless mesh networks," in CoNext. USA: ACM, 2007.

[9] — "Molecular architecture for autonomic wireless mesh networks," submitted, http://membres-liglab.imag.fr/theoleyre/uploads/molecular_ research_report.pdf, 2009.

[10] J. So and N. H. Vaidya, "Multi-channel mac for ad hoc networks: handling multi-channel hidden terminals using a single transceiver." in MOBIHOC. Japan: ACM, 2004.

[11] M. Heusse, F. Rousseau, R. Guillier, and A. Duda, "Idle sense: An optimal access method for high throughput and fairness in rate diverse wireless lans," in SIGCOMM. Philadelphia, USA: ACM, August 2005.

[12] J. E. Dunbar, J. W. Grossman, J. H. Hattingh, S. T. Hedetniemi, and A. A. McRae, "On weakly connected domination in graphs," Discrete Mathematics, vol. 167-168, 1997.

[13] H. M. K. Alazemi, A. Das, R. Vijaykumar, and S. Roy, "Fixed channel assignment algorithm for multi-radio multi-channel mesh networks," Wireless Communications and Mobile Computing, vol. 8, 2008.

[14] K. Jain, J. Padhye, V. Padmanabhan, and L. Qiu, "Impact of interference on multi-hop wireless network performance," in International Conference on Mobile Computing and Networking (MOBICOM). USA: ACM, September 2003.

[15] G. Chelius, A. Fraboulet, and E. Fleury, "Wsnet: a modular event-driven wireless network simulator," http://www.worldsens.net/, 2006.

[16] Coin-or branch and cut, "https://projects.coin-or.org/Cbc."

[17] V. Bahl, R. Chandra, and J. Dunagan, "SSCH: Slotted seeded channel hopping for capacity improvement in ieee 802.11 ad hoc wireless networks," in MOBICOM. USA: ACM, 2004.

[18] A. P. Subramanian, H. Gupta, and S. R. Das, "Minimum-interference channel assignment in multi-radio wireless mesh networks," in SECON. USA: IEEE, 2007. 